\title{
Nonlinear integral inequality with power and its application in delay integro-differential equations
}

\author{
Yazhou Tian ${ }^{1 *}$ and Min Fan²
}

"Correspondence:

tianyazhou369@163.com

'School of Mathematical Sciences,

University of Jinan, Jinan, P.R. China

Full list of author information is

available at the end of the article

\begin{abstract}
New nonlinear integral inequalities (NII) are presented in this paper. Based on mathematical analysis technique, several estimation results are obtained, which not only complement the aforementioned results, but also generalize the inequalities to the more general nonlinearities. As an application, they can be employed to estimate the bound on the solutions of power integro-differential equations (IDE).
\end{abstract}

MSC: 26D10; 26D15

Keywords: Nonlinear; Integral inequality; Power; Delay IDE

\section{Introduction}

As everyone knows, there exists a class of mathematical models described by differential equations, such as Malthus population model. However, a lot of differential equations do not possess the exact solution. Under this case, integral inequalities are significant for investigating the boundedness, stability, asymptotic behavior of solutions to differential equations. Gronwall [1] put forward the well-known Gronwall inequality to estimate the solution of linear differential equation. Bihari inequality [2] extended [1] to nonlinear one, and many authors have been devoted to studying NII in recent years [3-25]. For example, based on the generalized Gronwall inequality, Tian et al. [3] investigated the asymptotic behavior of switched delay systems that represent a class of systems in practical engineering and have wide application in automated highways, power systems, and so on. Pachpatte [4] considered a linear integral inequality (1.1).

Theorem 1.1 ([4]) Let $c_{0} \geq 0$ and $u, b, c, d \in C\left(R^{+}, R^{+}\right), R^{+}=[0,+\infty)$. If

$$
u(t) \leq c_{0}+\int_{0}^{t}(b(s) u(s)+d(s)) d s+\int_{0}^{t} b(s)\left(\int_{0}^{s} c(\xi) u(\xi) d \xi\right) d s,
$$

(c) The Author(s) 2020. This article is licensed under a Creative Commons Attribution 4.0 International License, which permits use, sharing, adaptation, distribution and reproduction in any medium or format, as long as you give appropriate credit to the original author(s) and the source, provide a link to the Creative Commons licence, and indicate if changes were made. The images or other third party material in this article are included in the article's Creative Commons licence, unless indicated otherwise in a credit line to the material. If material is not included in the article's Creative Commons licence and your intended use is not permitted by statutory regulation or exceeds the permitted use, you will need to obtain permission directly from the copyright holder. To view a copy of this licence, visit http://creativecommons.org/licenses/by/4.0/ 
then

$$
\begin{aligned}
u(t) \leq & c_{0}+\int_{0}^{t}\left[d(s)+b(s)\left(c_{0} \exp \left(\int_{0}^{s}(b(\sigma)+c(\sigma)) d \sigma\right)\right.\right. \\
& \left.\left.+\int_{0}^{s} d(\sigma) \exp \left(\int_{\sigma}^{s}(b(\tau)+c(\tau)) d \tau\right) d \sigma\right)\right] d s, \quad t \in R^{+} .
\end{aligned}
$$

After that, Abdeldaim and El-Deeb [12] generalized (1.1) and investigated the delay integral inequality (1.2).

Theorem 1.2 ([12, Theorem 2.1]) Assume that $c_{0} \geq 0, u, b, c, d \in C\left(R^{+}, R^{+}\right)$, and $\alpha \in$ $C^{1}\left(R^{+}, R^{+}\right)$are nondecreasing functions with $\alpha(t) \leq t, \alpha(0)=0$. If

$$
u(t) \leq c_{0}+\int_{0}^{\alpha(t)}(b(s) u(s)+d(s)) d s+\int_{0}^{\alpha(t)} b(s)\left(\int_{0}^{s} c(\xi) u(\xi) d \tau\right) d s,
$$

then

$$
\begin{aligned}
u(t) \leq & c_{0}+\int_{0}^{t}\left(\alpha^{\prime}(s) d(\alpha(s))+\alpha^{\prime}(s) b(\alpha(s)) \exp \left(\int_{0}^{\alpha(s)}(b(\xi)+c(\xi)) d \xi\right)\right. \\
& \left.\times\left(c_{0}+\int_{0}^{\alpha(s)} d(\sigma) \exp \left(-\int_{0}^{\sigma}(b(\xi)+c(\xi)) d \xi\right) d \sigma\right)\right) d s, \quad \forall t \in R^{+} .
\end{aligned}
$$

Very recently, Li and Wang [21] studied the power integral inequality (1.3).

Theorem 1.3 ([21, Theorem 2.1]) Suppose that $m, n, p \in(0,1]$ are nonnegative constants, $u, a, b, c \in C\left(R^{+}, R^{+}\right), \alpha \in C^{1}\left(R^{+}, R^{+}\right), \alpha(t)$ is nondecreasing with $\alpha(t) \leq t, \alpha(0)=0$. If

$$
u(t) \leq a(t)+\int_{0}^{\alpha(t)} b(s)\left(u^{m}(s)+\int_{0}^{s} c(\xi) u^{n}(\xi) d \xi\right)^{p} d s
$$

then

$$
u(t) \leq a(t)+A(t) \exp \left(\int_{0}^{\alpha(t)} p m b(s) d s+\int_{0}^{\alpha(t)} p b(s)\left(\int_{0}^{s} n c(\xi) d \xi\right) d s\right), \quad t \in R^{+},
$$

where

$$
\begin{aligned}
A(t)= & \int_{0}^{\alpha(t)} b(s)[(1-p)+p(m a(s)+(1-m))] d s \\
& +\int_{0}^{\alpha(t)} p b(s) \int_{0}^{s} c(\xi)[n a(\xi)+1-n] d \xi d s .
\end{aligned}
$$

Note that inequalities (1.2) and (1.3) have been proved in the cases $p=1$ and $p \in(0,1]$, respectively, how about $p>1$ ? The aforementioned results are not covered, and it would also be interesting to generalize the inequalities considered in $[4,12,21]$ to the more general nonlinearities, which is the motivation why we further study the above inequalities and their general cases.

We study some new power NII and establish several estimation results under the condition of $p>1$, which not only complement the ones established in $[4,12,21]$ but also 
generalize inequalities (1.1)-(1.3) to the more general nonlinearities. The obtained results can be employed to study the boundedness of the delay IDE. As an application, two illustrative examples are also presented.

\section{Main results}

Throughout the paper, $R=(-\infty,+\infty), R^{+}=[0,+\infty), C(D, E)$ and $C^{1}(D, E)$ defined on $D$ with range in $E$ are continuous functions and continuously differentiable function sets, respectively. The three lemmas are essential to proving the main results.

Lemma 2.1 ([21]) Let $a \geq 0$ and $m \geq n>0$. Then

$$
a^{n / m} \leq \frac{n}{m} a+\frac{m-n}{m}
$$

Lemma 2.2 ([25]) Assume that $u, v \geq 0$ and $p \geq 0$. Then

$$
(u+v)^{p} \leq K_{p}\left(u^{p}+v^{p}\right)
$$

where $K_{p}=1,0 \leq p \leq 1$, and $K_{p}=2^{p-1}, p>1$.

Lemma 2.3 Suppose that $p>0$ is a constant and $\alpha(t)$ is a nondecreasing function with $\alpha(t) \leq t, \alpha(0)=0, \alpha \in C^{1}\left(R^{+}, R^{+}\right), u, a, b, c, d \in C\left(R^{+}, R^{+}\right)$, and

$$
u(t) \leq a(t)+\int_{0}^{\alpha(t)} b(s)(c(s) u(s)+d(s))^{p} d s .
$$

Then

$$
u(t) \leq \begin{cases}a(t)+g(t)+\exp \left(\int_{0}^{\alpha(t)} h(s) d s\right) \int_{0}^{\alpha(t)} g(s) h(s) \exp \left(-\int_{0}^{s} h(\xi) d \xi\right) d s, & 0<p \leq 1, \\ a(t)+\left(k^{1-p}(t)+(1-p) \int_{0}^{\alpha(t)} 2^{p-1} b(s) c^{p}(s) d s\right)^{\frac{1}{1-p}}, & p>1,\end{cases}
$$

with

$$
k^{1-p}(t)>(p-1) \int_{0}^{\alpha(t)} 2^{p-1} b(s) c^{p}(s) d s
$$

where

$$
\begin{aligned}
& h(t)=p b(t) c(t), \\
& g(t)=\int_{0}^{\alpha(t)}[p b(s)(a(s) c(s)+d(s))+(1-p) b(s)] d s, \\
& k(t)=\int_{0}^{\alpha(t)} 2^{p-1} b(s)(a(s) c(s)+d(s))^{p} d s .
\end{aligned}
$$

Proof Define

$$
v(t)=\int_{0}^{\alpha(t)} b(s)(c(s) u(s)+d(s))^{p} d s .
$$


Then $v(t)$ is a nondecreasing function, and

$$
u(t) \leq a(t)+v(t)
$$

Therefore,

$$
v(t) \leq \int_{0}^{\alpha(t)} b(s)(c(s) v(s)+a(s) c(s)+d(s))^{p} d s .
$$

Next we will prove the following two cases $0<p \leq 1$ and $p>1$, respectively.

Case 1: $0<p \leq 1$.

By Lemma 2.1, we have

$$
(c(s) v(s)+a(s) c(s)+d(s))^{p} \leq p(c(s) v(s)+a(s) c(s)+d(s))+1-p .
$$

This combined with (2.5) yields

$$
\begin{aligned}
v(t) & \leq \int_{0}^{\alpha(t)} b(s)(c(s) v(s)+a(s) c(s)+d(s))^{p} d s \\
& \leq \int_{0}^{\alpha(t)}[p b(s)(c(s) v(s)+a(s) c(s)+d(s))+(1-p) b(s)] d s \\
& =g(t)+\int_{0}^{\alpha(t)} h(s) v(s) d s,
\end{aligned}
$$

where $h(t)$ and $g(t)$ are defined by (2.3). Define $J(t)=\int_{0}^{\alpha(t)} h(s) v(s) d s$, then $J(0)=0, J(t)$ is nondecreasing, $v(t) \leq g(t)+J(t)$, and

$$
\begin{aligned}
J^{\prime}(t) & =h(\alpha(t)) \alpha^{\prime}(t) v(\alpha(t)) \\
& \leq h(\alpha(t)) \alpha^{\prime}(t)(g(\alpha(t))+J(\alpha(t))) \\
& \leq h(\alpha(t)) g(\alpha(t)) \alpha^{\prime}(t)+h(\alpha(t)) \alpha^{\prime}(t) J(t),
\end{aligned}
$$

i.e.,

$$
J^{\prime}(t)-h(\alpha(t)) \alpha^{\prime}(t) J(t) \leq h(\alpha(t)) g(\alpha(t)) \alpha^{\prime}(t) .
$$

Multiplying (2.6) by $\exp \left(-\int_{0}^{\alpha(t)} h(s) d s\right)$ produces

$$
\begin{aligned}
& \exp \left(-\int_{0}^{\alpha(t)} h(s) d s\right)\left[J^{\prime}(t)-h(\alpha(t)) \alpha^{\prime}(t) J(t)\right] \\
& \quad \leq \exp \left(-\int_{0}^{\alpha(t)} h(s) d s\right) h(\alpha(t)) \alpha^{\prime}(t) g(\alpha(t)) .
\end{aligned}
$$

Integrating the above inequality from 0 to $t$, we have

$$
J(t) \leq \exp \left(\int_{0}^{\alpha(t)} h(s) d s\right) \int_{0}^{\alpha(t)} g(s) h(s) \exp \left(-\int_{0}^{s} h(\xi) d \xi\right) d s .
$$


Since $v(t) \leq g(t)+J(t)$, we have

$$
v(t) \leq g(t)+\exp \left(\int_{0}^{\alpha(t)} h(s) d s\right) \int_{0}^{\alpha(t)} g(s) h(s) \exp \left(-\int_{0}^{s} h(\xi) d \xi\right) d s .
$$

This together with (2.4) produces

$$
u(t) \leq a(t)+g(t)+\exp \left(\int_{0}^{\alpha(t)} h(s) d s\right) \int_{0}^{\alpha(t)} g(s) h(s) \exp \left(-\int_{0}^{s} h(\xi) d \xi\right) d s .
$$

Case 2: $p>1$.

Applying Lemma 2.2 to (2.5), we get

$$
\begin{aligned}
v(t) & \leq \int_{0}^{\alpha(t)} 2^{p-1} b(s)\left(c^{p}(s) v^{p}(s)+(a(s) c(s)+d(s))^{p}\right) d s \\
& =k(t)+\int_{0}^{\alpha(t)} 2^{p-1} b(s) c^{p}(s) \nu^{p}(s) d s,
\end{aligned}
$$

where $k(t)$ is defined by (2.3). Since $k(t)$ is a nondecreasing function, for fixed $T$,

$$
v(t) \leq k(T)+\int_{0}^{\alpha(t)} 2^{p-1} b(s) c^{p}(s) \nu^{p}(s) d s, \quad t \in[0, T] .
$$

Define

$$
w(t)=k(T)+\int_{0}^{\alpha(t)} 2^{p-1} b(s) c^{p}(s) v^{p}(s) d s .
$$

Then $w(0)=k(T), w$ is a nondecreasing function, and

$$
v(t) \leq w(t), \quad v(\alpha(t)) \leq w(\alpha(t)) \leq w(t) .
$$

Differentiating $w$ and using (2.7), we get

$$
\begin{aligned}
w^{\prime}(t) & =2^{p-1} \alpha^{\prime}(t) b(\alpha(t)) c^{p}(\alpha(t)) v^{p}(\alpha(t)) \\
& \leq 2^{p-1} \alpha^{\prime}(t) b(\alpha(t)) c^{p}(\alpha(t)) w^{p}(t)
\end{aligned}
$$

and

$$
\frac{w^{\prime}(t)}{w^{p}(t)} \leq 2^{p-1} \alpha^{\prime}(t) b(\alpha(t)) c^{p}(\alpha(t))
$$

The above inequality multiplied by $1-p$ gives

$$
(1-p) \frac{w^{\prime}(t)}{w^{p}(t)} \geq(1-p) 2^{p-1} \alpha^{\prime}(t) b(\alpha(t)) c^{p}(\alpha(t)) .
$$

By simple calculation of (2.8),

$$
w(t) \leq\left(k^{1-p}(T)+(1-p) \int_{0}^{\alpha(t)} 2^{p-1} b(s) c^{p}(s) d s\right)^{\frac{1}{1-p}}, \quad t \in[0, T] .
$$


Letting $t=T$ in the above inequality, we have

$$
w(T) \leq\left(k^{1-p}(T)+(1-p) \int_{0}^{\alpha(T)} 2^{p-1} b(s) c^{p}(s) d s\right)^{\frac{1}{1-p}}, \quad t \in[0, T] .
$$

Because $T$ is arbitrary,

$$
w(t) \leq\left(k^{1-p}(t)+(1-p) \int_{0}^{\alpha(t)} 2^{p-1} b(s) c^{p}(s) d s\right)^{\frac{1}{1-p}} .
$$

This together with (2.4), (2.7) implies

$$
u(t) \leq a(t)+\left(k^{1-p}(t)+(1-p) \int_{0}^{\alpha(t)} 2^{p-1} b(s) c^{p}(s) d s\right)^{\frac{1}{1-p}} .
$$

Based on Cases 1 and 2, we can draw a conclusion that $u(t)$ satisfies (2.2).

Theorem 2.1 Assume that $m, n, p$ are nonnegative constants satisfying $0<m, n \leq 1, p>1$, $\alpha(t)$ is nondecreasing with $\alpha \in C^{1}\left(R^{+}, R^{+}\right), \alpha(t) \leq t, \alpha(0)=0, u, a, b, c \in C\left(R^{+}, R^{+}\right)$, and

$$
u(t) \leq a(t)+\int_{0}^{\alpha(t)} b(s)\left(u^{m}(s)+\int_{0}^{s} c(\xi) u^{n}(\xi) d \xi\right)^{p} d s
$$

Then

$$
u(t) \leq a(t)+\left(\tilde{k}^{1-p}(t)+(1-p) \int_{0}^{\alpha(t)} 2^{p-1} b(s)\left(m+n \int_{0}^{s} c(\xi) d \xi\right)^{p} d s\right)^{\frac{1}{1-p}}
$$

with

$$
\tilde{k}^{1-p}(t)>(p-1) \int_{0}^{\alpha(t)} 2^{p-1} b(s)\left(m+n \int_{0}^{s} c(\xi) d \xi\right)^{p} d s
$$

where

$$
\tilde{k}(t)=\int_{0}^{\alpha(t)} 2^{p-1} b(s)\left(m a(s)+1-m+\int_{0}^{s} c(\xi)(n a(\xi)+1-n) d \xi\right)^{p} d s .
$$

Proof Construct

$$
y(t)=\int_{0}^{\alpha(t)} b(s)\left(u^{m}(s)+\int_{0}^{s} c(\xi) u^{n}(\xi) d \xi\right)^{p} d s .
$$

Then $y(0)=0, y$ is a nondecreasing function, and

$$
u(t) \leq a(t)+y(t) .
$$

By Lemma 2.1,

$$
\begin{aligned}
& (a(t)+y(t))^{m} \leq m(a(t)+y(t))+1-m, \\
& (a(t)+y(t))^{n} \leq n(a(t)+y(t))+1-n .
\end{aligned}
$$


From (2.11) and (2.12), we have

$$
\begin{aligned}
y(t) \leq & \int_{0}^{\alpha(t)} b(s)\left((a(s)+y(s))^{m}+\int_{0}^{s} c(\xi)(a(\xi)+y(\xi))^{n} d \xi\right)^{p} d s \\
\leq & \int_{0}^{\alpha(t)} b(s)\left(m(a(s)+y(s))+1-m+\int_{0}^{s} c(\xi)(n(a(\xi)+y(\xi))+1-n) d \xi\right)^{p} d s \\
= & \int_{0}^{\alpha(t)} b(s)\left[\left(m+n \int_{0}^{s} c(\xi) d \xi\right) y(s)\right. \\
& \left.+m a(s)+1-m+\int_{0}^{s} c(\xi)(n a(\xi)+1-n) d \xi\right]^{p} d s .
\end{aligned}
$$

Using Lemma 2.3,

$$
y(t) \leq\left(\tilde{k}^{1-p}(t)+(1-p) \int_{0}^{\alpha(t)} 2^{p-1} b(s)\left(m+n \int_{0}^{s} c(\xi) d \xi\right)^{p} d s\right)^{\frac{1}{1-p}}, \quad t \geq 0,
$$

where $\tilde{k}(t)$ is defined as in (2.10). This associated with (2.11) yields

$$
u(t) \leq a(t)+\left(\tilde{k}^{1-p}(t)+(1-p) \int_{0}^{\alpha(t)} 2^{p-1} b(s)\left(m+n \int_{0}^{s} c(\xi) d \xi\right)^{p} d s\right)^{\frac{1}{1-p}}
$$

Remark 2.1 When $0<p \leq 1$, inequality (2.9) has been studied in [12, Theorem 2.1] and [21, Theorem 2.1]. However, the above results cannot be applied to the case $p>1$. In Theorem 2.1, we further investigate (2.9) under the condition of $p>1$. To some extent, our result extends the results in [12, Theorem 2.1] and [21, Theorem 2.1].

Theorem 2.2 Suppose that $p, q, m, n$ are nonnegative constants with $q \geq m>0, q \geq n>0$, $p>0, \alpha(t)$ is nondecreasing with $\alpha \in C^{1}\left(R^{+}, R^{+}\right), \alpha(t) \leq t, \alpha(0)=0, u, a, b, c \in C\left(R^{+}, R^{+}\right)$, and

$$
u^{q}(t) \leq a(t)+\int_{0}^{\alpha(t)} b(s)\left(u^{m}(s)+\int_{0}^{s} c(\xi) u^{n}(\xi) d \xi\right)^{p} d s
$$

Then

$$
u(t) \leq \begin{cases}{\left[a(t)+\hat{g}(t)+\exp \left(\int_{0}^{\alpha(t)} \hat{h}(s) d s\right) \int_{0}^{\alpha(t)} \hat{g}(s) \hat{h}(s) \exp \left(-\int_{0}^{s} \hat{h}(\xi) d \xi\right) d s\right]^{1 / q},} & 0<p \leq 1 \\ {\left[a(t)+\left(\hat{k}^{1-p}(t)+(1-p) \int_{0}^{\alpha(t)} 2^{p-1} b(s)\left(\frac{m}{q}+\frac{n}{q} \int_{0}^{s} c(\xi) d \xi\right)^{p} d s\right)^{\frac{1}{1-p}}\right]^{1 / q},} & p>1\end{cases}
$$

with

$$
\hat{k}^{1-p}(t)>(p-1) \int_{0}^{\alpha(t)} 2^{p-1} b(s)\left(\frac{m}{q}+\frac{n}{q} \int_{0}^{s} c(\xi) d \xi\right)^{p} d s,
$$


where

$$
\begin{aligned}
\hat{h}(t)= & p b(t)\left(\frac{m}{q}+\frac{n}{q} \int_{0}^{t} c(\xi) d \xi\right), \\
\hat{g}(t)= & \int_{0}^{\alpha(t)}\left[p b ( s ) \left(\frac{m}{q} a(s)+\frac{q-m}{q}\right.\right. \\
& \left.\left.+\int_{0}^{s} c(\xi)\left(\frac{n}{q} a(\xi)+\frac{q-n}{q}\right) d \xi\right)+(1-p) b(s)\right] d s, \\
\hat{k}(t)= & \int_{0}^{\alpha(t)} 2^{p-1} b(s)\left(\frac{m}{q} a(s)+\frac{q-m}{q}+\int_{0}^{s} c(\xi)\left(\frac{n}{q} a(\xi)+\frac{q-n}{q}\right) d \xi\right)^{p} d s .
\end{aligned}
$$

Proof Construct

$$
z(t)=\int_{0}^{\alpha(t)} b(s)\left(u^{m}(s)+\int_{0}^{s} c(\xi) u^{n}(\xi) d \xi\right)^{p} d s
$$

Then $z(0)=0, z$ is a nondecreasing function, and

$$
u(t) \leq(a(t)+z(t))^{1 / q} .
$$

By Lemma 2.1,

$$
\begin{aligned}
& u^{m}(t) \leq(a(t)+z(t))^{m / q} \leq \frac{m}{q}(a(t)+z(t))+\frac{q-m}{q}, \\
& u^{n}(t) \leq(a(t)+z(t))^{n / q} \leq \frac{n}{q}(a(t)+z(t))+\frac{q-n}{q} .
\end{aligned}
$$

From (2.16)-(2.18),

$$
\begin{aligned}
z(t) \leq & \int_{0}^{\alpha(t)} b(s)\left((a(s)+z(s))^{m / q}+\int_{0}^{s} c(\xi)(a(\xi)+z(\xi))^{n / q} d \xi\right)^{p} d s \\
\leq & \int_{0}^{\alpha(t)} b(s)\left(\frac{m}{q}(a(s)+z(s))+\frac{q-m}{q}+\int_{0}^{s} c(\xi)\left(\frac{n}{q}(a(\xi)+z(\xi))+\frac{q-n}{q}\right) d \xi\right)^{p} d s \\
= & \int_{0}^{\alpha(t)} b(s)\left[\left(\frac{m}{q}+\frac{n}{q} \int_{0}^{s} c(\xi) d \xi\right) z(s)\right. \\
& \left.+\frac{m}{q} a(s)+\frac{q-m}{q}+\int_{0}^{s} c(\xi)\left(\frac{n}{q} a(\xi)+\frac{q-n}{q}\right) d \xi\right]^{p} d s
\end{aligned}
$$

Applying Lemma 2.3 to (2.19), we can obtain

$$
z(t) \leq \begin{cases}\hat{g}(t)+\exp \left(\int_{0}^{\alpha(t)} \hat{h}(s) d s\right) \int_{0}^{\alpha(t)} \hat{g}(s) \hat{h}(s) \exp \left(-\int_{0}^{s} \hat{h}(\xi) d \xi\right) d s, & 0<p \leq 1, \\ \left(\hat{k}^{1-p}(t)+(1-p) \int_{0}^{\alpha(t)} 2^{p-1} b(s)\left(\frac{m}{q}+\frac{n}{q} \int_{0}^{s} c(\xi) d \xi\right)^{p} d s\right)^{\frac{1}{1-p},} & p>1,\end{cases}
$$

where $\hat{h}(t), \hat{g}(t)$, and $\hat{k}(t)$ are defined by (2.15). This associated with (2.17) yields (2.14).

Remark 2.2 Inequality $(2.13)$ generalizes the ones in $[4,12,21]$ to the more general nonlinear case. 


\section{Examples}

Now, we study the boundedness of the integral equation and IDE with delay.

Example 3.1 Consider the Volterra type integral equation with delay

$$
x(t)=a(t)+\int_{0}^{\alpha(t)} b(s)\left(x(s)+\int_{0}^{s} c(\xi) x(\xi) d \xi\right)^{3} d s, \quad t \in R^{+},
$$

where $a, b, c \in C(R, R), \alpha \in C^{1}\left(R^{+}, R^{+}\right), \alpha(t) \leq t, \alpha(0)=0, \alpha(t)$ is a nondecreasing function. Then (3.1) satisfies

$$
|x(t)| \leq|a(t)|+\int_{0}^{\alpha(t)}|b(s)|\left(|x(s)|+\int_{0}^{s}|c(\xi)||x(\xi)| d \xi\right)^{3} d s .
$$

Let $u(t)=|x(t)|$ and rewrite (3.2):

$$
u(t) \leq|a(t)|+\int_{0}^{\alpha(t)}|b(s)|\left(u(s)+\int_{0}^{s}|c(\xi)| u(\xi) d \xi\right)^{3} d s .
$$

By Theorem 2.1,

$$
u(t) \leq|a(t)|+\left(\tilde{k}^{-2}(t)-8 \int_{0}^{\alpha(t)}|b(s)|\left(1+\int_{0}^{s}|c(\xi)| d \xi\right)^{3} d s\right)^{-\frac{1}{2}}
$$

with

$$
\tilde{k}(t)<\frac{\sqrt{2}}{4}\left(\int_{0}^{\alpha(t)}|b(s)|\left(1+\int_{0}^{s}|c(\xi)| d \xi\right)^{3} d s\right)^{-\frac{1}{2}},
$$

where

$$
\tilde{k}(t)=4 \int_{0}^{\alpha(t)}|b(s)|\left(|a(s)|+\int_{0}^{s}|c(\xi)||a(\xi)| d \xi\right)^{3} d s,
$$

which illustrates that the solution of (3.1) is bounded.

Example 3.2 Consider the delay IDE

$$
\left(x^{q}(t)\right)^{\prime}=F\left(t, x(\tau(t)), \int_{0}^{t} G(\xi, x(\tau(\xi))) d \xi\right),
$$

and $x(t)=\varphi(t), t \in[d, 0]$ with $-\infty<d=\inf \{\tau(t), t \in I\} \leq 0, \tau(t) \leq t$, where $x(t)$ and $x(\tau(t))$ are the state and state delay, respectively. $F \in C\left(R^{+} \times R \times R, R\right)$ and $G \in C\left(R^{+} \times R, R\right)$ satisfy

$$
\begin{aligned}
& |F(t, U, V)| \leq b(t)\left(|U|^{m}+|V|\right)^{p}, \\
& |G(t, W)| \leq c(t)|W|^{n}, \quad t \in R^{+},
\end{aligned}
$$

where $b, c \in C\left(R^{+}, R^{+}\right), q \geq m>0, q \geq n>0, p>0$. Integrating (3.3) produces

$$
x^{q}(t)=x^{q}(0)+\int_{0}^{t} F\left(s, x(\tau(s)), \int_{0}^{s} G(\xi, x(\tau(\xi))) d \xi\right) d s .
$$


Letting $u(t)=|x(t)|$, then

$$
\begin{aligned}
u^{q}(t) & \leq u^{q}(0)+\int_{0}^{t} b(s)\left(u^{m}(\tau(s))+\int_{0}^{s} c(\xi) u^{n}(\tau(\xi)) d \xi\right)^{p} d s \\
& \leq|\varphi(t)|^{q}+\int_{0}^{\tau(t)} \frac{b\left(\tau^{-1}(s)\right)}{\tau^{\prime}\left(\tau^{-1}(s)\right)}\left(u^{m}(s)+\int_{0}^{s} \frac{c\left(\tau^{-1}(\xi)\right)}{\tau^{\prime}\left(\tau^{-1}(\xi)\right)} u^{n}(\xi) d \xi\right)^{p} d s .
\end{aligned}
$$

Employing Theorem 2.2 to (3.4) produces the following: when $0<p \leq 1$,

$$
u(t) \leq\left[|\varphi(t)|^{q}+\hat{g}(t)+\exp \left(\int_{0}^{\alpha(t)} \hat{h}(s) d s\right) \int_{0}^{\alpha(t)} \hat{g}(s) \hat{h}(s) \exp \left(-\int_{0}^{s} \hat{h}(\xi) d \xi\right) d s\right]^{1 / q},
$$

where

$$
\begin{aligned}
\hat{h}(t)= & p \frac{b\left(\tau^{-1}(t)\right)}{\tau^{\prime}\left(\tau^{-1}(t)\right)}\left(\frac{m}{q}+\frac{n}{q} \int_{0}^{t} \frac{c\left(\tau^{-1}(\xi)\right)}{\tau^{\prime}\left(\tau^{-1}(\xi)\right)} d \xi\right), \\
\hat{g}(t)= & \int_{0}^{\tau(t)}\left[p \frac { b ( \tau ^ { - 1 } ( s ) ) } { \tau ^ { \prime } ( \tau ^ { - 1 } ( s ) ) } \left(\frac{m}{q}|\varphi(s)|^{q}+\frac{q-m}{q}\right.\right. \\
& \left.\left.+\int_{0}^{s} \frac{c\left(\tau^{-1}(\xi)\right)}{\tau^{\prime}\left(\tau^{-1}(\xi)\right)}\left(\frac{n}{q}|\varphi(\xi)|^{q}+\frac{q-n}{q}\right) d \xi\right)+(1-p) \frac{b\left(\tau^{-1}(s)\right)}{\tau^{\prime}\left(\tau^{-1}(s)\right)}\right] d s,
\end{aligned}
$$

when $p>1$,

$$
\begin{aligned}
u(t) \leq & \left\{|\varphi(t)|^{q}+\left[\hat{k}^{1-p}(t)\right.\right. \\
& \left.\left.+(1-p) \int_{0}^{\tau(t)} 2^{p-1} \frac{b\left(\tau^{-1}(s)\right)}{\tau^{\prime}\left(\tau^{-1}(s)\right)}\left(\frac{m}{q}+\frac{n}{q} \int_{0}^{s} \frac{c\left(\tau^{-1}(\xi)\right)}{\tau^{\prime}\left(\tau^{-1}(\xi)\right)} d \xi\right)^{p} d s\right]^{\frac{1}{1-p}}\right\}^{1 / q}
\end{aligned}
$$

with

$$
\hat{k}^{1-p}(t)>(p-1) \int_{0}^{\tau(t)} 2^{p-1} \frac{b\left(\tau^{-1}(s)\right)}{\tau^{\prime}\left(\tau^{-1}(s)\right)}\left(\frac{m}{q}+\frac{n}{q} \int_{0}^{s} \frac{c\left(\tau^{-1}(\xi)\right)}{\tau^{\prime}\left(\tau^{-1}(\xi)\right)} d \xi\right)^{p} d s,
$$

where

$$
\begin{aligned}
\hat{k}(t)= & \int_{0}^{\tau(t)} 2^{p-1} \frac{b\left(\tau^{-1}(s)\right)}{\tau^{\prime}\left(\tau^{-1}(s)\right)}\left(\frac{m}{q}|\varphi(s)|^{p}+\frac{q-m}{q}\right. \\
& \left.+\int_{0}^{s} \frac{c\left(\tau^{-1}(\xi)\right)}{\tau^{\prime}\left(\tau^{-1}(\xi)\right)}\left(\frac{n}{q}|\varphi(\xi)|^{p}+\frac{q-n}{q}\right) d \xi\right)^{p} d s .
\end{aligned}
$$

\section{Acknowledgements}

The authors sincerely thank the editor and anonymous reviewers for their careful reviews and useful suggestions on improving the presentation of the paper.

\section{Funding}

This work was supported by the National Natural Science Foundation of China under grant nos. 61807015, 61703180, the Natural Science Foundation of Shandong Province (ZR2017LF012), A Project of Shandong Province Higher Educational Science and Technology Program (J17KA157), and the Doctoral Scientific Research Foundation of University of Jinan (1008398) 


\section{Competing interests}

The authors declare that they have no competing interests.

\section{Authors' contributions}

The authors contributed equally to this work. They both read and approved the final version of the manuscript.

\section{Author details}

${ }^{1}$ School of Mathematical Sciences, University of Jinan, Jinan, P.R. China. ${ }^{2}$ Qingdao University of Technology, Feixian, P.R. China.

\section{Publisher's Note}

Springer Nature remains neutral with regard to jurisdictional claims in published maps and institutional affiliations.

\section{Received: 1 November 2019 Accepted: 20 March 2020 Published online: 31 March 2020}

\section{References}

1. Gronwall, T.H.: Note on the derivatives with respect to a parameter of the solutions of a system of differential equations. Ann. Math. 20, 292-296 (1919)

2. Bihari, I.: A generalization of a lemma of Bellman and its application to uniqueness problems of differential equations. Acta Math. Acad. Sci. Hung. 7, 71-94 (1956)

3. Tian, Y.Z., Cai, Y.L., Sun, Y.G.: Asymptotic behavior of switched delay systems with nonlinear disturbances. Appl. Math. Comput. 268, 522-533 (2015)

4. Pachpatte, B.G.: Inequalities for Differential and Integral Equations. Academic Press, London (1998)

5. Zada, A., Ali, W., Park, C.: Ulam's type stability of higher order nonlinear delay differential equations via integral inequality of Gronwall-Bellman-Bihari's type. Appl. Math. Comput. 350, 60-65 (2019)

6. Jiang, F.C., Meng, F.W.: Explicit bounds on some new nonlinear integral inequalities with delay. J. Comput. Appl. Math. 205, 479-486 (2007)

7. Feng, Q.H., Meng, F.W., Zheng, B.: Gronwall-Bellman type nonlinear delay integral inequalities on time scales. J. Math. Anal. Appl. 382, 772-784 (2011)

8. Gu, J., Meng, F.W.: Some new nonlinear Volterra-Fredholm type dynamic integral inequalities on time scales. Appl. Math. Comput. 245, 235-242 (2014)

9. Liu, H.D.: A class of retarded Volterra-Fredholm type integral inequalities on time scales and their applications. J. Inequal. Appl. 2017, Article ID 293 (2017)

10. Liu, H.D.: Some new integral inequalities with mixed nonlinearities for discontinuous functions. Adv. Differ. Equ. 2018 Article ID 22 (2018)

11. Li, W.N.: Some new dynamic inequalities on time scales. J. Math. Anal. Appl. 319, 802-814 (2006)

12. Abdeldaim, A., El-Deeb, A.A.: On generalized of certain retarded nonlinear integral inequalities and its applications in retarded integro-differential equations. Appl. Math. Comput. 256, 375-380 (2015)

13. Tian, Y.Z., Fan, M., Meng, F.W.: A generalization of retarded integral inequalities in two independent variables and their applications. Appl. Math. Comput. 221, 239-248 (2013)

14. Fan, M., Tian, Y.Z., Meng, F.W.: A class of dynamic integral inequalities with mixed nonlinearities and their applications in partial dynamic systems time scales. Adv. Differ. Equ. 2019, Article ID 12 (2019)

15. Tian, Y.Z., El-Deeb, A.A., Meng, F.W.: Some nonlinear delay Volterra-Fredholm type dynamic integral inequalities on time scales. Discrete Dyn. Nat. Soc. 2018, Article ID 5841985 (2018)

16. Agarwal, R.P., Deng, S., Zhang, W.: Generalization of a retarded Gronwall-like inequality and its applications. Appl. Math. Comput. 165, 599-612 (2005)

17. Saker, S.H.: Applications of Opial inequalities on time scales on dynamic equations with damping terms. Math. Comput. Model. 58, 1777-1790 (2013)

18. Xu, R., Meng, F.W.: Some new weakly singular integral inequalities and their applications to fractional differential equations. J. Inequal. Appl. 2016, Article ID 78 (2016)

19. Xu, R., Ma, X.T.: Some new retarded nonlinear Volterra-Fredholm type integral inequalities with maxima in two variables and their applications. J. Inequal. Appl. 2017, Article ID 187 (2017)

20. Wang, W.S., Zhou, X.L., Guo, Z.H.: Some new retarded nonlinear integral inequalities and their applications in differential-integral equations. Appl. Math. Comput. 218, 10726-10736 (2012)

21. Li, Z.Z., Wang, W.S.: Some nonlinear Gronwall-Bellman type retarded integral inequalities with power and their applications. Appl. Math. Comput. 347, 839-852 (2019)

22. Boudeliou, A.: On certain new nonlinear retarded integral inequalities in two independent variables and applications. Appl. Math. Comput. 335, 103-111 (2018)

23. Wan, L.L., Xu, R.: Some generalized integral inequalities and there applications. J. Math. Inequal. 7, 495-511 (2013)

24. Luo, Y.Y., Xu, R.: Some new weakly singular integral inequalities with discontinuous functions for two variables and their applications. Adv. Differ. Equ. 2019, Article ID 387 (2019)

25. Hilderbrandt, T.H.: Introduction to the Theory of Integration. Academic Press, New York (1963) 